sank next morning from $15 t$ to 128 , and was firmer; respiration had risen from 32 to 42 .

On the following morning, we found that our patient had passed a bad night, and had had much delirium. She felt very ill. The pulse was 112 , steady, though very feeble; respiration 40. A catheter was required. One ounce of brandy was ordered to be given every two hours. She expressed herself as feeling better in the evening; her voice was firmer and louder; there. was no delirium.

On the day following, her state was satisfactory; she continued to take an ounce of brandy every two hours. But next day she was not so well; she had wandered more, though her appetite had returned. The amount of brandy had been somewhat lessened; the original quantity was therefore restored, and quinine was prescribed.

The brandy was continued for one or two days. On the eighth day of my attendance, the dulness had gone, and vesicular breathing was restored; but her pulse and respiration continued 130 and 40 respectively. She speedily recovered sufficiently to be removed to her mother's.

I must add that, in this case, I thought the patient's condition nearly desperate; I had little hope of a favourable termination of the case. Nor can I entertain a doubt, that the successful issue could have been procured by no other means than by the free administration of brandy which was employed. As I do not vaunt the use of stimulants as a certain cure for pneumonia, I think it unnecessary to occupy further time by detailing cases in which these remedies failed to save the patient. I only wish freely to admit that such cases have occurred to me, but that they presented not the smallest reason to warrant the supposition that the stimulants were concerned in producing the fatal event.

I wish to state, in conclusion, that I desire to extend the remarks I have now applied to pneumonia, so as to embrace the whole subject of inflammation; admitting the same limitations, and advocating a similar plan of administering stimulants, when occasion appears to call for their use.

\section{OBSERVATIONS ON THE PHYSIOLOGICAL ACTION OF ACONITINA.*}

By Dyce Duckworth, Esq., Student in Medicine in the University of Eidinburgh.

THE special physiological actions of aconitina have re. ceived able attention and observation at the hands of Drs. Fleming and Headland. Upon reading over carefully the different kinds of action said to be exerted by this body, I was particularly struck with a singular incongruity in the accounts as to the condition of the pupil, both during the time when the poison was acting, and also after death had resulted. This fact, amongst others, led me to make a series of experiments with the alkaloid, which extended over a period of five months, and were performed whenever leisure and circumstances permitted. The first object was to obtain pure aconitina ; for I imagined that possibly the incongruous statements regarding its action might in some measure, at least, be due to the employment of impure samples of the alkaloid. From its costliness, it is apt to be adulterated, or met with in an impure condition. I therefore obtained three samples-one prepared by Messrs. Smith of Edin. burgh; another by Mr. Morson of London; and a third foreign one, which was not quite pure. I conld rely upon the purity of the first two samples, by applying the only test known for the alkaloid, viz., confidence in the

* Excernt from a Dissertation upon the Physiological and The rapeutical Actions of the Alkaloids. Read before the Royal Medical Society of Edinburgh, December 14th, 1860. characters of the chemists who prepared and sold it; and $I$ found them equally efficacious and infinitely superior to the foreign specimen.

I experimented upon rabbits, cats, and kittens, internally; and topically upon the conjunctivæ of two human adults.

Action of Aconitina given internally. When aconitina in solution is given by the mouth, the first effect pro- $\bar{\nabla}$ duced is a most interse tingling sensation in the tongue $\varrho$ and fauces: this is followed instantly by a copious secretion of saliva, over which the patient appears to lose control, since it is not swallowed, but flows over the lips in a $\vec{\circ}$ frothy and slimy condition. This is seen to the greatest excess in the case of the cat; not nearly so much is $\vec{\omega}$ secreted in the rabbit. Dr. Headland thinks this condi. O tion is due in some measure to paralysis of the fances. In the human subject, the patient is observed to clutch 3 . at the throat after aconite-poisoning; and all animals exhibit uneasiness in that region, as if to assist or $c$ accomplish deglutition, which function seems to be lost iN in all cases, while pharyngeal and cosophageal actions $N$ appear rather to he reversed. The respiratory furctions are next apparently interfered with; the breathing becomes laboured and spasmodic; and cries are uttered $N$ with more or less vigour, according to the amount of the dose. Next vomiting occurs, and may continue several times; this is attributed, with good reason, by Headland, $\frac{\rho}{\supset}$ to derangement of the function of the vagus, since post $\overrightarrow{.}$ mortem examination reveals no appreciable change in $\infty$ the coats of the stomach. Sensation next appears en- $\rightarrow$ tirely lost; and the animal, as if mad, tosses itself about wildly; endeavours to walk, but cannot; falls over on its side; takes prodigious leaps; and is then prostrated. Convulsions generally appear at this time, and the breathing becomes slower and more difficult. Finally, total paralysis is observable, and death ensues.

Condition of the Pupil. During the action of the $\stackrel{\mathbb{Q}}{\AA}$ poison, the pupils are slightly contracted; but I have $\overrightarrow{\vec{B}}$ noticed in all cases that, about two or three minutes be- $\overline{\bar{O}}$ fore death, and at the time of death, they ailate enor- 3 mously, and generally continue so after death, though $I ?$ observed in some cases that, in an hour or two after death, one pupil contracted, while the other remained unchanged; and I have even seen the contraction vary in the same pupil several times within twelve hours after death. The action on the pupil differs, however, according to the manner in which the poison is introduced into the system. I find the prevailing opinion to be, that aconite causes contraction; Headland gives it $ᄋ$ as his opinion, that dilatation is produced. It is a very difficult matter to say what the real effect is. Dr. Fleming states, in his almirable dissertation on Aconite (p.11), that " in general the pupil is more or less con- N tracted, dilating to its natural size immediately on the $\sigma$ cessation of respiration". This statement is the most correct one I have met with, though I differ from it in that I hold the pupils to dilate considerably beyond $\mathrm{N}$ their normal size at the time of death; in fact, this was a most constant and striking symptom in every case in which I employed sufficierit to cause death. Hence $I_{O}$ would say, as a rule, the pupil is more or less contracted, dilating enormously a few moments before and at the time of death.

As to the cause of this, Fleming states that the symptoms seem to him attributable to the same pathological cause as the convulsions. He attributed the convulsions to cerebral congestion, since they only occurred when the poison acted slowly, and the respirations were diminished; so that, from advancing asphyxia, $\overrightarrow{\mathbb{D}}$ the venous system must have been highly congested. "Contraction was present in all the cases in which con vulsions occurred." I can verify this latter statement. He found that, when muriate of aconitina was injectedo into the veins, the pupils dilated before any amount of venous congestion could have taken place (and I haveo 
always observed that dilatation occurs speedily when a large dose is given, which acts fatally soon); bence he concluded that dilatation is the specific or direct effect on the pupil.

We must believe that dilatation ensues upon cerebral congestion; and our main difficulty, it seems, is to account for the subsequent final dilatation after contraction: this, I conceive, we must trace to purely cerebral influences.

We shall refer to this subject again in considering the manner in which aconitina causes death; but I may give briefly a case of poisoning by aconite which happened this year, and which has been most minutely communicated to me through the kindness of Dr. Nevins, of Liverpool. He writes, "I had a patient, a young lady, who swallowed a fluiddrachm of the officinal tincture of aconite. ... For about six hours her life was in imminent danger. She recovered at last, however. When first seen by her father, an hour after taking it, the pupils were so dilated that no iris could be seen, and this condition lasted till the following day. It was a fortnight before she had entirely lost the tingling and numbness, and feeling of malaise, and for weeks after she was subject to sudden fits of numbness in the feet, so severe as to cause her to fall, even six months afterwards she was liable to vomitings and sensations of cramp in the throat, to which she was not subject before she took the poison." This was a very remarkable case. One of the most marked features in poisoning by aconite in the human subject, and which I have not met with in any treatises on the matter, is, the total inability to act or speak, though consciousness is to a considerable extent maintained up to the time of death; the mind, too, is often peculiarly active.

Patients describe their sensations as if their tongues were gummed to their mouth; and they could give no alarm as to their condition. The eyes are kept wide open; and there is confusion of vision, sometimes with inability to close the eyes at the command of the will.

Topical Action of Aconitina. When aconitina is ap. plied to the skin in solution or in the form of ointment, it quickly paralyses the superficial sensory nerves, causing the usual benumbing sensation. I tried topical application of about one-fortieth of a grain of the alkaloil mixed with water to the human conjunctiva, in two cases. Slight congestion of the membrane occurred, and photophobia in botb cases; also slight contraction of the pupil. The tingling sensations continued for six hours; and at the end of that time the eyeball, in one case, protruded an eighth of an inch from the socket; in the other, the tingling was referred to the angle of the jaw and down the side of the neck.

These were severe experiments and not to be repeated; they further lost an entire night's rest to both patients. Now the contraction which was induced in these cases was attributable, not to any specific action of the alka. loid, but to irritation produced mechanically by its fine particles.

Dr. Fleming is of this opinion, and we know that dust and irritants of all kinds induce contraction when directly applied to the eye. Fleming found that when aconitina ointment was applied to the conjunctiva contraction occurred speedily, which lasted for several hours; but when it was applied to the forehead or temple the pupil became occasionally dilated and partial blinduess ensued-this he attributes to reflex action through the third and fifth nerves.

I applied one-thirtieth of a grain of pure aconitina in ointment between the eyes of a cat, but, as this animal possesses evident volition over the iris, it was impossible to state with any confidence the pupillary state afterwards; in two hours time the animal was foaming at the mouth and not breathing quite normally, also suffering from general malaise; she had probably been cleaning herself and may have licked her paws.
It is clear that we can never make use of aconitina as a direct local therapeutic agent for the eye.

Mode in which Aconitina causes Death. This is a question which can hardly be called settled. Some authorities attribute death to asphyxia; others, including Dr. Headland, to cardiac syncope.

The subject was brought before the British Association at Oxford last year by Dr. E. R. Harvey; he performed his experiments upon dogs, rabbits, and frogs with Fleming's tincture of aconite; and he considers they went to prove that aconite acts first on the nerves, and of then on the muscles, killing by its action on the heart; the blood and urine, he stated, were found normal. In the discussion on this paper, Sir B. Brodie remarked that in his experiments many years ago with the expressed juice of aconite, he found it to act as a narcotic, and to arrest respiration; and he recommended artificial respiration for recovery from the effects. Dr. Sharpey said that in some experimerts of his, he had noticed the same effects as Dr. Harvey; viz., the stoppage of the heart's action, and of muscular irritability, the nerves $N$ also leading to the affected muscles were deprived of $\oplus$ their excitability. Thus Drs. Harvey and Sharpey attri- 음 bute death to syncope, and Sir B. Brodie to asphyxia. Professor Bennett remarks, in his Principles and Practice of Medicine, on a case of aconite poisoning, that " it is difficult to separate the effects of syncope from those of asphyxia in such a case, since the first condition $\supset$ must induce the other." Fleming states that three $\vec{\infty}$ varieties are observable in the symptoms and mode of $\infty$ death : first, it may prove fatal by a powerfully sedative. inpression on the nervous system; secondly, by suspension of the respiratory functions; and thirdly, by syncope; he further remarks that the latter conditions are always more or less conjoined.

My own observations have led me to the following conclusions. When a large dose of aconitina is given, $I \frac{\mathrm{O}}{\mathbb{1}}$ think death is caused speedily either by direct cardiac syncope, or by a powerfully sedative impression on the nervous system, and certainly the action of the heurt ceases. In this case there are no convulsions as in death from asphyxia, and the dilated pupils may here be the direct and perhaps specific effect of aconitina when it? acts so speedily as to prevent any amount of cerebral congestion.

When a small dose is employed, death is averted for a time, the heart's action is enfeebled by degrees, so that 0 the pulmonary and systemic circulation go on very 3 slowly. Meantime, the phrenic and respiratory system of nerves are becoming affected; respirations are per-응 formed less frequently, and are accompanied with deep $₹$ spasmodic action of the diaphragm, which will also ac- 응 count for the cries uttered, the laryngeal muscles being paralysed; the venous system becomes engorged, and $N$ the pupils dilate finally from cerebral congestion, while the heart and lungs cease to act. In this case, I consider, a combination of asphyxia and syncope causes death. In both cases sensibility is soon lost, and mus- N cular paralysis is observable early. The poison may be N classed as a general sedative.

The Pith of the Pine and its Syrup. M. Lagapeco has concentrated the pith of the pinus maritima into a pectoral syrup, which has been used with much benefit in many chronic diseases, such as catarrh, humid asthma, bronchorrhœa; it has also been used successfully in the $T$ acute indispositions of winter, such as rheumatism, laryngitis, pharyngitis, angina, etc. This syrup of the $\mathbb{D}$ pith of pine has also an evident action on the urinary $\frac{C}{\mathbb{D}}$ secretions and inflammatory irritation of the bladder. 2 It advantageously does away with the other medicinal $\sigma$ preparations from the pine which were so generally used upon the introduction of this the most essen. O tial and most intimate part of the tree. (Revue Médicale.) 